\title{
Las revistas de psicología en Chile: historia y situación actual
}

\section{Psychology Journals in Chile: history and current situation}

\author{
Roberto Polanco-Carrasco \\ Asociación Chilena de Revistas Científicas de Psicología, Chile \\ Miguel Gallegos \\ Consejo Nacional de Investigaciones Científicas y Técnicas, Argentina. Universidad Nacional de Rosario, Argentina \\ Gonzalo Salas \\ Universidad Católica del Maule, Chile \\ Wilson López-López \\ Universidad Javeriana, Colombia
}

Rec (31 de enero de 2017) Acept (28 de febrero de 2017)

\begin{abstract}
Resumen
El presente artículo expone una revisión del desarrollo histórico y estado actual de las publicaciones científicas seriadas en psicología en Chile. Para determinar el análisis de las revistas se realizó una recuperación de las mismas por medio de su solicitud de ISSN (International Standard Serial Number), lo cual arrojó un resultado de 39 revistas clasificadas en la categoría psicología. De la cantidad total de revistas, 14 siguen vigentes y la mitad de ellas se encuentran indizadas en bases de datos como Web of Science, Scopus, SciELO y Redalyc. Se concluye que una mayor preocupación por potenciar los índices bibliométricos y cienciométricos de las revistas, permitirá mejorar las indizaciones de las revistas de psicología y de esta forma contribuir al mejoramiento de la calidad, visibilidad e impacto de estas tanto en Chile como en el resto de Latinoamérica. Palabras clave: Revistas de Psicología, Psicología en Chile, Historia de la Psicología, Trabajo editorial, comunicación científica.
\end{abstract}

\begin{abstract}
This article is a review of the historical and current state of the periodical scientific publications about psychology in Chile. In order to choose the journals, a search of these journals was made through the ISSN (International Standard Serial Number), which found 39 journals classified in the category of "psychology". From the total number of journals, 14 are still being published and half of them are indexed in databases such as Web of Science, Scopus, SciELO and Redalyc. Based on this analysis, it is possible to conclude that it is necessary to promote and enhance bibliometric and scienciometric indexes of journals, which will improve the indexing of psychology journals, and thus contribute to the improvement of the quality, visibility and impact of these magazines, both in Chile and the rest of Latin America.

Key words: Psychology Journals, Psychology in Chile, History of Psychology, Editorial work, scientific communication.
\end{abstract}

\footnotetext{
* Correspondencia: Roberto Polanco-Carrasco, Revista Cuadernos de Neuropsicología Panamerican Journal of Neuropsychology, Valparaíso 396, Rancagua (Chile). E-mail: polanco.roberto@gmail.com
} 


\section{Introducción}

El escenario científico actual se encuentra atravesando por un tiempo de cambios profundos en las formas de producir, socializar y transferir el conocimiento generado en los claustros académicos (Gibbons et. al., 1997; Naidorf, 2009). En gran medida, las nuevas dinámicas del conocimiento científico han tenido un impacto importante en el quehacer tradicional de los investigadores, al mismo tiempo que han planteado nuevos desafíos para las instituciones académicas respecto de sus políticas, sus metas y la sustentabilidad económica de sus proyectos (Gallegos, Berra, Benito y López López, 2014). Además se ha vuelto necesario repensar el marco crítico desde el cual se enfocan las investigaciones y la forma en la que se abordan los objetos de conocimiento, para posibilitar respuestas más adecuadas a los problemas sociales contemporáneos (Montenegro, Pujol y VargasMonroy, 2015).

Uno de los cambios más visibles de la práctica investigativa se encuentra relacionada con la publicación de los resultados, los cuales se exigen que sean difundidos en revistas con buenos índices de impacto, lo que redundaría en mayores grados de visibilidad y aprovechamiento de los conocimientos generados. Por supuesto que este panorama también presenta mayores exigencias para los investigadores, máxime si se encuentran en un contexto científico considerado periférico (Cueto, 1989; Vessuri, 1983), ya que deben procurar los mejores medios para someter a evaluación sus productos. Al mismo tiempo, el trabajo editorial se ha visto trastocado por la presión de mejorar la indexación y categorización de las revistas científicas, lo que ha favorecido una mayor profesionalización de las ediciones científicas en general (López-López, 2014; Polanco-Carrasco, 2016b).

Algunos países de América Latina y el Caribe han implementado importantes cambios en materias de visibilidad y difusión de la producción científica en la región, sin embargo los datos indican que los países latinoamericanos representan solamente el $2 \%$ de la inversión mundial en Investigación y Desarrollo (I+D), muy lejos de lo ocurrido en Norteamérica, Europa y Asia (Valderrama, 2012); por lo tanto, se requiere un avance sustantivo y un cambio paradigmático para lograr de indicadores que puedan estar a la altura y así posicionar de mejor forma a nuestros países frente los más desarrollados. De hecho, al estudiar los mayores países productores de open access sólo aparece Brasil en los 22 primeros (Miguel, Tannuri de Oliveira y Cabrini, 2016). Sin embargo, se valora la creación de bases de datos, portales de revistas y acceso abierto a los artículos académicos. Al respecto se han organizado diversos encuentros, foros y congresos de especialistas, al mismo tiempo que se ha proporcionado una importante cantidad de literatura en estos temas (Calleja y Albornoz, 2006; Cetto, 2009; Cetto y Hillerud, 1995; Cetto y Alonso, 1999; Haupt, 2000; LunaMorales y Collazo-Reyes, 2007; Miguel, 2011; Montoya, 2012). Todas estas acciones implementadas desde la década de 1990 han dado importantes frutos y han permitido dimensionar más adecuadamente los déficits y potenciales con los que cuenta la región, pero aún se espera mayores y mejores resultados; sobre todo en materia de colaboración, aspecto que se comentó tempranamente al referir la relevancia del trabajo organizado por los investigadores (Newcomb, 1906; Sherrington, 1922) y que paulatinamente fue adquiriendo fuerza al avanzar los indicadores bibliométricos que consideran la coautoría como un aspecto relevante de evaluar, para así visibilizar la colaboración nacional e internacional de los autores y organizaciones (Subramanyan, 1983; García, Acevedo-Triana y López-López, 2014).

Desde luego, el campo disciplinar de la psicología no ha sido ajeno a todo este acontecer, dado que también se realizaron varios cónclaves motivados por la necesidad de mejorar la indexación de las revistas académicas y aunar esfuerzos para potenciar la visibilidad de las producciones locales (Aguado-López, Becerril-García y Aguilar, 2016; Buela-Casal y López López, 2005; Cardoso Sampaio, 2008; Cardoso Sampaio y Zoqui Paulovic, 2012; Corredor Aristizábal, 2016; García Martínez y Guerrero Bote, 2008; López-López, 2010, 2015; Pérez-Acosta, 2003; Polanco-Carrasco, 2015, 2016a; Trzesniak, Plata-Caviedes y Córdoba-Salgado, 2012; VandenBos y Winkler, 2015; Zych y Buela-Casal, 2010). Algunos países como Argentina, Brasil, Chile, Colombia y México desarrollaron políticas específicamente diseñadas para alentar el mejoramiento de la visibilidad de las producciones y la indexación de las revistas de psicología (Alonso Olivas-Ávila, Musi-Lechuga, Quevedo-Blasco y Luna-Hernández, 2012; Ávila-Toscano, Marenco-Escuderos y Madariaga, 2014; Bizarro, 2006; Fin Motta, Gomes de Castro y Pizzinato, 2015; Gamba, Packer y Meneghini, 2015; Gómez-Morales, Jaraba-Barrios, Guerrero-Castro y López-López, 2012; López-López, de Moya Anegón, AcevedoTriana, Garcia, y Silva, 2015; Quevedo-Blasco y López López, 2010, 2011; Vera-Villaroel, López López, Lillo y Silva, 2011).

En este trabajo se analiza el estado de situación de las revistas chilenas de psicología, contemplando aquellas publicaciones seriadas que han obtenido su código numérico normalizado de identificación, el ISSN (International Standard Serial Number). Como se sabe, el ISSN consiste en un código de identificación único que permite identificar 
formalmente a las publicaciones periódicas de todo el mundo. Desde 1982 el Consejo Nacional de Investigación Científica y Tecnológica (CONICYT) es el encargado de habilitar los registros de las revistas periódicas en Chile. Para conocer el número de las revistas de psicología editadas en el país se realizó una consulta de las solicitudes de ISSN de acuerdo a los siguientes criterios: Psicología como Materia y Ciencias Sociales como Disciplina. De las publicaciones de psicología pesquisadas, se logró recuperar un número 39 de revistas seriadas. A partir del conjunto de las publicaciones localizadas se realizó el seguimiento de cada una para identificar qué publicaciones siguen vigentes y cuáles han dejado de editarse. También se examinó los diversos formatos de publicación, las modalidades de suscripción y la indexación de las revistas. Todo este análisis fue contextualizado por una breve historización de las publicaciones periódicas en psicología en América Latina.

\section{Breve historia de las publicaciones periódicas en ciencia}

Una parte importante del trabajo científico involucra, además de la propia labor de investigación, la socialización de los conocimientos producidos, ya sea entre la propia comunidad de pares como en los ámbitos sociales más generalizados. Para cumplir con el propósito de difundir los resultados de las investigaciones se han instituido las publicaciones científicas periódicas, cuya estructura se diferencia de otras publicaciones periódicas, como los diarios y las revistas de divulgación, al incorporar la evaluación del contenido antes de ser publicado, tarea que es llevada a cabo por pares expertos en la modalidad de doble ciego frecuentemente.

En la historia de la ciencia, las primeras revistas científicas datan del siglo XVII, cuando se organiza el Journal des Savants, en 1665, en Francia, el Philosophical Transactions, en 1665, en Inglaterra, el Litteratti de Italia, en 1668, en Italia, y Miscellanea Curiosa, en 1670, en Alemania, entre otras. Por su parte, en América Latina se considera al Mercurio Volante, editado en México a partir de 1772, como la primera revista médica de todo el continente americano (López, 2000). Tales publicaciones se originaron como parte de las actividades de las nacientes sociedades científicas constituidas por esas épocas y fueron concebidas para dar lugar a la comunicación especializada, reemplazando poco a poco a los libros como medios de difusión científica predilecta, todo lo cual permitió la organización de la ciencia moderna tal como hoy la conocemos. En sus inicios, las revistas científicas copiaron el modelo de los periódicos informativos, en la que se incluían editoriales, cartas, estudios y críticas. Con los años, y el consecuente aumento de la producción científica, se hizo más frecuente la modalidad de artículos y reportes breves de investigación, con la respectiva citación de las referencias bibliográficas, lo cual permitió dar crédito a los autores, su propiedad intelectual y la originalidad de los estudios (Price, 1973).

En el caso particular de la psicología, los primeros canales de difusión fueron la revista Mind, creada por Alexander Bain en 1876, en Inglaterra (Brown, 2005; Staley, 2009), el Philosophische Studien, instituido por Wilhelm Wundt en 1881, en Alemania (Titchener, 1921), The American Journal of Psychology, fundado por Granville Stanley Hall en 1887 (Fuchs, 2012; Green y Feinerer, 2015, 2016; Stanley Hall y Titchener, 1921), en Estados Unidos, el Zeitschrift für Psychologie und Physiologie der Sinnesorgane, creado por Hermann Ebbinghaus y Arthur Konig en 1890, en Alemania (Woodworth, 1909), la L>Année Psychologique, establecida por Henri Beaunies y Alfred Binet en 1894 en Francia (Delabarre, 1895), y el Psychological Review, fundado por James Mark Baldwin y James McKeen Cattell en 1894 en Estados Unidos (Catell, 1898; Green, Feinerer y Burman, 2015).

Si bien la historia de las publicaciones periódicas en psicología tiene su origen en estas revistas hacia fines del siglo XIX, con el transcurrir del nuevo siglo se promoverá una difusión más generalizada de las publicaciones seriadas en todo el mundo. Tal expansión se explica por la importante consolidación de la psicología como ciencia y profesión. En efecto, durante el siglo XX la psicología no solo logra consolidarse como una disciplina de conocimiento con estricto rigor científico, sino además se transforma en una profesión capaz de resolver innumerables problemas de la sociedad contemporánea (Gallegos, 2014a).

En América Latina, ya desde los primeros años del siglo XX, la difusión de los conocimientos psicológicos se propició en diversas revistas afines a la disciplina, tales como las publicaciones de pedagogía, criminología, neurología, sociología o filosofía. No obstante, también desde las primeras décadas se organizaron algunas publicaciones especializadas en la disciplina, por ejemplo, los Anales de Psicología de la Sociedad de Psicología, de los cuales se conocieron 3 tomos entre 1910 y 1914, y donde se volcaron la mayoría de los estudios realizados por los miembros de dicha sociedad que comenzó a funcionar en 1909 en Buenos Aires. Tiempo después, esa incipiente publicación fue recuperada por la edición de los Anales del Instituto de Psicología, de la Facultad de Filosofia y Letras, de la Universidad de Buenos Aires, los cuales se convirtieron en 
la primera revista de alcance regional, ya que se publicaron trabajos de autores latinoamericanos (Ardila, 1986; Sanz Ferramola y Klappenbach, 2000).

En Rio de Janeiro, el polaco Waclaw Radecki reunió varios de sus estudios en Trabalhos de Psychologia, cuya publicación solo se efectuó en 1928 y 1929 (Centofanti, 1982). Unas décadas más tarde se editaron los Arquivos Brasileiros de Psicotécnica, en 1949, luego reconvertidos en Arquivos Brasileiros de Psicologia Aplicada, a partir 1969, para devenir finalmente como Arquivos Brasileiros de Psicologia, desde 1979 hasta la actualidad (Jacó-Vilela, 1999). En Uruguay se publicó un pequeño boletín intitulado Hoja de Psicología, el cual comenzó a editarse en 1947. En sus páginas se difundieron las actividades desarrolladas por el Centro de Estudios Psicológicos de Montevideo, y cuya organización estuvo a cargo del mismo Radecki que había estado en Brasil unas décadas atrás (León 1997, 2014). En Lima tuvo lugar la edición del Boletín del Instituto Psicopedagógico Nacional, donde el alemán Walter Blumenfeld publicó varias de sus investigaciones (Alarcón, 2000). En Chile, las revistas propiamente psicológicas tuvieron un desarrollo tardío, por lo cual dichas temáticas eran publicadas en revistas de ciencias de carácter generalista como El Crepúsculo, periódico literario y científico, fundado en 1843 (Salas, 2013), Anales de la Universidad de Chile, fundado en 1844 (Cruz, 2011) y posteriormente Atenea, fundada en 1924 en la Universidad de Concepción, que si bien era una revista cultural propiciaba el desarrollo de trabajos académicos y científicos (Nascimento, 1924), o bien en revistas de educación, higiene, entre otras.

Entre los años 1920 y 1922 se publicó la revista Mente, órgano central del Instituto Central de Ciencia Armonía fundado en 1912 por Georgina Hooper de Mandiola. La revista era su órgano de difusión y si bien no fue una revista científica, sino todo lo contrario, la intención declarada de sus miembros era exponer los principios de la "ciencia mental" para promover la concentración del pensamiento y desarrollo de la voluntad (Salas, 2015).

Abelardo Iturriaga fue el organizador de los Archivos del Instituto de Psicología, en la Universidad de Chile, en 1941, donde básicamente se publicaron las investigaciones que se realizaban en dicha institución (Kaulino, 2016; Ligüeño y Parra, 2007; Parra, 2015).

En su conjunto, todas estas iniciales publicaciones sirvieron para difundir los conocimientos psicológicos generados en los laboratorios e institutos de psicología que se fueron generando durante la primera mitad del siglo XX en América Latina. Lamentablemente muchas de aquellas revistas tuvieron una vida efímera, salvo muy pocas excepciones. Además se trata de publicaciones que fueron concebidas en un tiempo donde todavía no se habían organizado las carreras de psicología, y por tanto, aun no existían psicólogos formados en la región, más allá de los que habían obtenido una especialización en el exterior o habían emigrado a estas latitudes. Con la organización de las primeras carreras de psicología hacia mediados del siglo XX comienza otra historia de las publicaciones periódicas en psicología en la región: Chile, 1947, Colombia, 1948, Guatemala, 1950, Brasil, 1953, Argentina, 1954, Perú, 1955, Venezuela, 1956 y México, 1959 (Alarcón, 2004; Ardila, 1986; Gallegos, 2009; Klappenbach y Pavesi, 1994).

A partir de la creación de estos primeros programas de formación académica se inicia el periodo de la profesionalización psicológica, y con ella se da paso a una nueva organización de las actividades científicas y profesionales. Además de organizarse colegios profesionales, sociedades científicas y diversas actividades académicas, también se inician nuevos proyectos editoriales en materia de publicaciones periódicas, muchos de las cuales van a ser proyectados desde la esfera institucional de las carreras de psicología y serán gestionados por los propios titulados en psicología. Por citar algunos ejemplos que todavía perduran, la Revista Mexicana de Psicología, comenzó sus actividades en 1984, más allá de la publicación de varios números de una revista de nombre homónimo en 1963 (Ardila, 1986; Lafarga, 1984). Una situación similar aconteció con la Revista Cubana de Psicología, la cual empezó a editarse en 1984, siendo que ya existía un precedente de 1955 (Cairo Valcárce, 2000; Gallegos, en prensa), por su parte, la Revista Argentina de Psicología inició sus actividades en 1969 y todavía continua editándose (Klappenbach y Arrigoni, 2011). La Revista Colombia de Psicología, que se edita desde 1992, reemplazó las actividades editoriales de la antigua Revista de Psicología, publicada de forma irregular entre 1956 y 1991 (Anónimo, 1991; López Caicedo, 1992).

Desde luego, estas revistas no fueron los únicos emprendimientos editoriales, en todos los países se puede encontrar una infinidad de publicaciones periódicas en psicología, aunque la mayoría ha tenido una vida muy corta. Tal es el caso de la Revista Chilena de Psicología y la Revista Uruguaya de Psicología, las cuales se editaron desde 1978, sin tener una periodicidad regular. En Perú se editaron numerosas publicaciones periódicas (Alarcón, 2000), pero solo algunas llegaron a tener una buena regularidad como la Revista de Psicología, de la Pontificia Universidad Católica del Perú, iniciada en 1983 (Blumen, 2013), y la revista Liberabit, de la Universidad San Martin de Porres, que ya cuenta con más de 20 años de edición (Romero Croce, 2014). Otras 
publicaciones que han logrado mantenerse con buena regularidad son la Revista Costarricense de Psicología desde 1982 (Carvajal y Matamoros, 2012; Céspedes Alvarado, 2008), y la Revista Puertorriqueña de Psicología desde 1983 (Martínez-Taboas y Pérez Pedrogo, 2006).

En la historia de las publicaciones seriadas en psicología también es preciso mencionar que muchas de las mismas fueron promovidas por estudiantes de psicología. En efecto, se ha constatado que los estudiantes de psicología en América Latina fueron activos protagonistas de la gestión de diversas revistas de psicología en casi todos los países de la región (Arbaiza-Bayona, 2012; Gallegos, 2009, 2014b; García, 2016). Incluso algunas revistas estudiantiles se transformaron en la publicación oficial de los programas de psicología, como en el caso de Cuadernos de Psicología, publicados desde 1959 hasta 1962 por la Asociación de Estudiantes de Psicología, de la Escuela de Psicología, de la Universidad Central de Venezuela. En otros casos, las publicaciones de los estudiantes asumieron un rol crítico y cuestionador respecto de la formación profesional, las corrientes teóricas en psicología y el perfil social que debía desarrollar la psicología en los países de la región (Gallegos, 2009, 2014b).

En el amplio repertorio de las revistas periódicas, es igualmente necesario describir aquellos emprendimientos editoriales que surgieron con el objetivo de volverse una publicación de alcance regional, como en el caso de la Revista Interamericana de Psicología, la cual comenzó a editarse desde 1967, bajo los auspicios de la Sociedad Interamericana de Psicología (Cassepp, 2004; Colotla, 2015; Gallegos, 2013). De igual forma, es necesario referir la importante trayectoria de la Revista Latinoamericana de Psicología, fundada en 1969 por Rubén Ardila (Gallegos, 2010; Gutiérrez, Pérez-Acosta y Plata-Caviedes, 2009). Estas importantes revistas, que hoy se siguen editando, representan esos exiguos ejemplos que se conocen en la región respecto de publicaciones periódicas de larga duración y edición ininterrumpida, además de mantener buenos estándares de calidad. Otra importante revista de alcance regional fue Acta Psiquiátrica y Psicológica de América Latina, creada en 1954 como Acta Neuropsiquiátrica Argentina, luego reconvertida en Acta Psiquiátrica y Psicológica Argentina en 1962, para luego adquirir su actual denominación desde 1964 (Gutiérrez, Pérez-Acosta y Plata-Caviedes, 2009).

No está de más indicar que en el contexto iberoamericano varias revistas editadas en España no solo fueron importantes órganos de difusión del conocimiento psicológico generado por los autores latinoamericanos, sino además fueron publicaciones que permitieron tomar contacto con los avances en la disciplina en idioma castellano (Bregman, López López y García, 2015; Carbonell y Calvó, 2009; Marín, 1985; Quevedo-Blasco, 2013, 2015; Quevedo-Blasco y López López, 2011; Vera-Villaroel, López López, Lillo y Silva, 2011). Quizás la de mayor envergadura sea la Revista de Psicología General y Aplicada, fundada en 1946 (Carpintero y Tortosa, 1996), aunque también Anuario de Psicología, creado en 1969 (Beyés, 2015; Guárdia Olmos, 2015), Anales de Psicología, iniciado en 1984 (Peñaranda Ortega, Quiñones Vidal y López García, 2005; Romero Medina, 2009), y Psicothema, organizada en 1989 (Quevedo-Blasco y Ariza, 2013). Desde luego, también se gestionaron varias publicaciones en diferentes áreas de actividad psicológica. Por solo citar las de alcance regional, la Revista de la Asociación Latinoamericana de Psicología Social, en 1981, Avances en Psicología Clínica Latinoamericana, en 1982, Revista Interamericana de Psicología Ocupacional, en 1982, Archivo Latinoamericano de Historia de la Psicología y Ciencias Afines, en 1988, y la Revista Iberoamericana de Diagnóstico y Evaluación Psicológica, en 1995, entre otras.

En la actualidad sigue proliferando la edición de nuevas revistas periódicas en psicología y otras van alcanzado buenos estándares de calidad editorial. Una lista completa de las mismas podría ser innecesaria ya que existen diversos portales de revistas, como Latindex, Scielo, Redalyc y Dialnet donde se las puede recuperar fácilmente. No obstante, vale mencionar que muchas de las publicaciones periódicas en psicología han obtenido la inclusión en importantes bases de datos y otras se encuentran en el camino de lograrlo, lo que pone en evidencia una política editorial sostenida en el tiempo, la cual se acompaña de un trabajo editorial con diversos grados de profesionalización. En lo que sigue, nos concentraremos en destacar el estado de situación de las revistas de psicología en Chile, dejando pendiente un análisis más detallado para el resto de los países de América Latina.

\section{Las revistas chilenas de psicología}

La profesionalización de la psicología en Chile comienza a partir de 1947 cuando Carlos Nassar formalizó el primer programa de formación de psicólogos en el país, en el marco de la Universidad de Chile (Laborda y Quezada, 2010; Luco, 2010; Salas, 2014; Salas y Lizama, 2013). Una década más tarde fue formalizada la segunda Escuela de Psicología, en la Pontificia Universidad Católica de Chile, en 1958 (Bravo, 2004). A partir de la década de 1980 comienza un progresivo crecimiento de instituciones de educación superior que ofrecerán la carrera de psicología (Urzúa, 2008; Urzúa, Vera-Villarroel, Zúñiga y Salas, 2015). Con el periodo de 
profesionalización se inicia un nuevo ciclo de las publicaciones periódicas en psicología en el país. Si bien antes de la organización de los primeros programas se conocieron varias revistas seriadas que incorporaron los conocimientos psicológicos en sus páginas, con la apertura de las carreras de psicología y el explosivo incremento de programas de formación se van a desenvolver más contundentemente los oficios editoriales en la psicología chilena. En la Tabla 1 se puede visualizar el número total de revistas de psicología que registraron su solicitud de código de identificación ISSN en el país, señalando como disciplina Ciencias Sociales y materia Psicología. Como se puede observar, más de una publicación puede presentar nombres similares o idénticos, pero cada una tendrá un ISSN exclusivo.

El periplo editorial indica que la primera revista de psicología catastrada durante el periodo profesional en Chile, acontece luego de transcurridos más de 30 años de iniciada la profesión psicológica en el país. En efecto, Cuadernos de Psicología, a cargo de la Universidad de Chile, editó su primer número a mediados de 1972, pero lamentablemente solo llegaron a publicarse pocos volúmenes. Ya se ha comentado que el Colegio de Psicólogos de Chile, organizado en 1968 para velar por los aspectos gremiales de la profesión, fue responsable de la edición de la Revista Chilena de Psicología, cuya actividad tuvo una extensa continuidad hasta que finalmente fue discontinuada de manera abrupta luego de publicar el primer número del volumen 24 el año 2003. A los fines históricos, debe mencionarse que dicha institución también contaba con un Boletín de Psicología, el cual fue publicado a partir de $1968 \mathrm{con}$ el fin de divulgar cuestiones relacionadas con la profesión del psicólogo en Chile. No se desconoce que los Archivos del Instituto de Psicología, que iniciaron su publicación en 1941, fueron reactivados sin mayor éxito en una segunda época en 1963. Cabe aclarar que aun cuando el boletín y la edición de los archivos en la segunda época pueden ser considerados como publicaciones efectuadas durante el periodo profesional, las mismas no fueron incluidas en la Tabla 1 debido a que

Tabla 1. Revistas Chilenas de Psicología

\begin{tabular}{|c|c|c|c|}
\hline Año & Título & Institución & ISSN \\
\hline 1972 & Cuadernos de Psicología & Universidad de Chile & 0378-097X \\
\hline 1978 & Revista Chilena de Psicología & Colegio de Psicólogos de Chile & 0716-3630 \\
\hline 1982 & Terapia Psicológica & Sociedad Chilena de Psicología Clínica & $\begin{array}{l}0716-6184 \\
0718-4808\end{array}$ \\
\hline 1984 & Psicosexualidad & Instituto de Desarrollo Psicológico I & 0716-1492 \\
\hline 1988 & Psicología y Ciencias Humanas & Universidad Central de Chile & 0716-6761 \\
\hline 1990 & Revista de Psicología & Universidad de Chile & 0716-8039 \\
\hline 1991 & Estudios Psicológicos & Universidad de La Frontera & 0716-8403 \\
\hline 1992 & Psykhe & Pontificia Universidad Católica de Chile & $\begin{array}{l}0717-0297 \\
0718-2228\end{array}$ \\
\hline 1993 & De Familias y Terapias & Instituto Chileno de Terapia Familiar & $0717-0173$ \\
\hline 1999 & In-Fieri & Universidad José Santos Ossa & $0717-4233$ \\
\hline 1999 & Praxis & Universidad Diego Portales & $0717-473 X$ \\
\hline 1999 & Castalia & Universidad Academia de Humanismo Cristiano & $0717-4985$ \\
\hline 2000 & Gradiva & Sociedad Chilena de Psicoanalítica y Psicoanálisis & $0717-6600$ \\
\hline 2000 & Límite & Universidad de Tarapacá & $\begin{array}{l}0718-1361 \\
0718-5065 \\
\end{array}$ \\
\hline 2001 & Revista de Psicología & Universidad de Valparaíso & 0717-604X \\
\hline 2001 & Puercoespín & Universidad Bolivariana & $0717-6937$ \\
\hline 2002 & Pensamiento \& Sociedad & Universidad Central de Chile & $0717-7658$ \\
\hline 2002 & Psicoperspectivas & Pontificia Universidad Católica de Valparaíso & \begin{tabular}{|l|}
$0717-7798$ \\
$0718-6924$ \\
\end{tabular} \\
\hline 2003 & Summa Psicológica & Universidad Santo Tomás & $0718-0446$ \\
\hline 2004 & Babel & Universidad Bolivariana & \begin{tabular}{|l|}
$0718-0187$ \\
$0718-6614$
\end{tabular} \\
\hline
\end{tabular}




\begin{tabular}{|c|c|c|c|}
\hline 2004 & Enlace Psicología & Opening Ltda & $0718-1558$ \\
\hline 2004 & Resonancias & Universidad del Mar & $0718-1647$ \\
\hline 2006 & Revista Chilena de Psicología Clínica & Sin información & $\begin{array}{l}0718-3666 \\
0718-3674 \\
\end{array}$ \\
\hline 2006 & Revista Chilena de Neuropsicología & Universidad de la Frontera & $0718-4913$ \\
\hline 2007 & Cuadernos de Neuropsicología & Centro de Estudios Académicos en Neuropsicología & $0718-4123$ \\
\hline 2007 & Ciencia Psicológica & Universidad Central de Chile & $0718-4166$ \\
\hline 2008 & Psicología Organizacional Humana & Universidad Adolfo Ibáñez & $0718-5626$ \\
\hline 2008 & Revista Chilena de Integración en Psicología & Sin información & 0718-6010 \\
\hline 2008 & Icimnag & INPAVCT S.A. & $0718-6665$ \\
\hline 2009 & Homo Sacer & Pontificia Universidad Católica de Valparaíso & 0718-6827 \\
\hline 2009 & Cosmogonías & Pontificia Universidad Católica de Valparaíso & 0718-722X \\
\hline 2009 & Salud \& Sociedad & $\begin{array}{l}\text { Universidad Católica del Norte } \\
\text { Universidad de Talca } \\
\text { Universidad de Tarapacá }\end{array}$ & $0718-7475$ \\
\hline 2009 & Cogency & Universidad Diego Portales & $0718-8285$ \\
\hline 2011 & Revista Pequén & Universidad del Bío-Bío & 0719-112X \\
\hline 2011 & Sujeto, Subjetividad y Cultura & Universidad de Artes y Ciencias Sociales & 0719-1553 \\
\hline 2011 & Revista de Psicología & Universidad de Viña del Mar & 0719-188X \\
\hline 2012 & Liminales & Universidad Central de Chile & $\begin{array}{l}0719-1758 \\
0719-7748 \\
\end{array}$ \\
\hline 2012 & Revista Latinoamericana de Psicología Social Ignacio Martín Baró & Universidad Alberto Hurtado & $0719-2703$ \\
\hline 2014 & Revista Latinoamericana de Psicología Positiva & Asociación Chilena de Psicología Positiva & $0719-4420$ \\
\hline
\end{tabular}

Nota. Los casos en que cuenten con 2 ISSN, el segundo corresponde a la versión online.

no poseían sus respetivos ISSN. De cualquier manera, hay que situarlas como parte del acervo histórico de la edición de revistas periódicas en psicología en Chile.

Con la entrada de la década de 1980 se inician nuevos proyectos editoriales, los cuales se asumen como publicaciones no generalistas, sino más bien especializadas como Terapia Psicológica, en el ámbito de la psicología clínica y Psicosexualidad, en el área que indica su nombre, sumado a Psicología y Ciencias Humanas, con un perfil marcadamente interdisciplinario. Desde el punto de vista histórico, los años ochenta representan el tiempo en el que se produce un acelerado crecimientos de nuevos programas de formación en psicología en Chile (Toro y Villegas, 1999). Si hasta ese tiempo solo existían dos programas en todo el país, en los años posteriores el número se iría incrementando sobremanera hasta llegar a los 139 según los últimos registros (Urzúa, Vera-Villarroel, Zúñiga y Salas, 2015). De alguna manera, la proliferación de las instituciones formadoras de psicólogos y el crecimiento de diversas sociedades de psicología vienen a explicar el aumento de las revistas de psicología periódicas en el país, ya que ellas serán las encargadas de gestionar nuevas publicaciones seriadas. En la Tabla 2 se puede cotejar la evolución de las revistas de psicología en Chile según el año de creación.

Tabla 2. Crecimiento de las Revistas Chilenas de Psicología en décadas

\begin{tabular}{|c|c|c|c|c|}
\hline 1970 & 1980 & 1990 & 2000 & 2010 \\
\hline 2 & 3 & 7 & 21 & 6 \\
\hline
\end{tabular}

La primera década del siglo XXI fue la que concentró la mayoría de los emprendimientos editoriales en materia de revistas periódicas. Solo en esa década se fundaron más revistas que en el conjunto de las otras cuatro décadas consignadas. Lógicamente el crecimiento de las publicaciones periódicas en psicología se encuentra correlacionado con el incremento poblacional de los estudiantes y titulados en psicología en el país (Toro y Villegas, 1999; Urzúa, VeraVillarroel, Zúñiga y Salas, 2015). Este incremento poblacional de psicólogos a nivel nacional se ve acompañado de un aumento en el número de investigaciones en psicología clínica, sin embargo esta producción todavía se encuentra limitada esencialmente a unas pocas instituciones (Vera-Villarroel, y Lillo, 2006). Un rasgo notorio en las publicaciones de esta primera década es que si bien la gestación de revistas 
generalistas no desapareció, la tendencia se orientó hacia la organización de publicaciones periódicas en diferentes áreas de la psicología. Probablemente este rasgo sea similar a lo acontecido en otros países de la región, donde también se ha destacado la creación de revistas temáticas en lugar de las revistas generalistas.

Más allá del entusiasmo y la multiplicación desmesurada de revistas periódicas de psicología en Chile, el sostenimiento de las mismas no logró ser lo más satisfactorio en el tiempo. Muchos de los emprendimientos que se proyectaron en el pasado ya no están vigentes. Algunas apenas alcanzaron a editar un único número, mientras que otras tuvieron una edición muy irregular, desembocando indefectiblemente en su desaparición. En la Tabla 3 se registran las revistas de psicología que todavía se siguen editando en Chile.

De las 14 revistas periódicas que todavía se editan en Chile, la mitad pueden ser consideradas revistas que publican trabajos en todas las áreas de la psicología, mientras que la otra mitad se especializa en un área determinada. Dos revistas se especializan en el ámbito neuropsicológico (Revista Chilena de Neuropsicología y Cuadernos de Neuropsicología), dos más en el área clínica (Terapia Psicológica y De Familias y Terapias), una que asume su espacio entre la psicología de la salud y la psicología social (Salud y Sociedad) y otra que se centra en la interdisciplinariedad entre filosofía y psicología (Limite). Por supuesto, varias de las revistas generalistas han promovido diversos números temáticos a lo largo de su historia editorial. La mayoría de estas publicaciones es editada desde la esfera institucional universitaria, es decir, son gestionadas por alguna escuela o departamento involucrado en la formación de psicólogos. El resto de las revistas son promocionadas por grupos o sociedades científicas.

Si bien lo habitual en las revistas de psicología en Chile es el acceso abierto sin cobro por leer ni publicar, se registran algunos casos tales como las revistas De Familia y Terapias y Cogency que cobran por el acceso a los artículos, ya sea por la modalidad de suscripción a la revista o bien por cada artículo individual. Se tiene conocimiento de otras tres revistas que si bien no poseen ISSN, no obstante, han sido recuperadas en este estudio. Por una parte, Cuadernos de Psicología Jurídica, cuya edición corre a cargo desde el 2004 por la Asociación Chilena de Psicología Jurídica y Forense, también encontramos MIME.SIS la revista digital de la Facultad de Ciencias Sociales de la Universidad de las Américas y la revista de la Escuela de Psicología de la Universidad del Pacifico Visión Posible. Por otra parte, se encuentra Psicología Hoy, publicación de la Universidad Alberto Hurtado, la cual se concibe como una publicación de divulgación y se encuentra plenamente vigente desde el año 2010. Quizá existan otras publicaciones periódicas en la psicología chilena que no han sido alcanzadas por los parámetros definidos en este estudio, por ejemplo las

Tabla 3. Revistas de psicología editadas en la actualidad

\begin{tabular}{llll}
\hline Año & Título & Institución & ISSN \\
\hline 1982 & Terapia Psicológica & Sociedad Chilena de Psicología Clínica & $0716-6184$ \\
$0718-4808$ & $0716-8039$ \\
\hline 1990 & Revista de Psicología & Universidad de Chile & $0717-0297$ \\
\hline 1992 & Psykhe & Pontificia Universidad Católica de Chile & $0718-2228$ \\
\hline 1993 & De Familias y Terapias & Instituto Chileno de Terapia Familiar & $0717-0173$ \\
\hline 1999 & Praxis & Universidad Diego Portales & $0717-473 X$ \\
\hline 1999 & Castalia & Universidad Academia de Humanismo Cristiano & $0717-4985$ \\
\hline 2000 & Limite & Universidad de Tarapacá & $0718-1361$ \\
\hline 2002 & Psicoperspectivas & Pontificia Universidad Católica de Valparaíso & $0717-7798$ \\
\hline 2003 & Summa Psicológica & Universidad Santo Tomás & $0718-6924$ \\
\hline 2006 & Revista Chilena de Neuropsicología & Universidad del Desarrollo & $0718-0446$ \\
\hline 2007 & Cuadernos de Neuropsicología & Centro de Estudios Académicos en Neuropsicología & $0718-0551$ \\
\hline 2009 & Salud \& Sociedad & Universidad Católica del Norte & $0718-4913$ \\
\hline 2009 & Cogency & Universidad Diego Portales & $0718-4123$ \\
\hline 2014 & Revista Latinoamericana de Psicología Positiva & Asociación Chilena de Psicología Positiva & $0718-7475$ \\
\hline
\end{tabular}

Nota Se consideran vigentes aquellas revistas que se editaron regularmente hasta la primera mitad del 2016. 
revistas estudiantiles de psicología, no obstante, cualquier otro estudio en el futuro podrá complementar este registro.

\section{La Asociación Chilena de Revistas Cientificas de Psicología}

Las dificultades detectadas y compartidas por un grupo de editores de psicología y la consecuente necesidad de mejorar la labor editorial de las revistas periódicas en psicología fue el pretexto para conformar una asociación que permitiera elaborar y proyectar una política para las revistas seriadas de psicología en Chile. A tal efecto se constituyó la Asociación Chilena de Revistas Científicas de Psicología, en octubre de 2009, siendo electos como su primer presidente el editor de Cuadernos de Neuropsicología, Roberto Polanco-Carrasco y secretaria la editora de Psicoperspectivas, Verónica López. Desde esa fecha, las acciones llevadas a cabo por la sociedad consistieron fundamentalmente en proporcionar asesoramiento a los editores responsables y sus equipos editoriales, con el objetivo de volver más profesional la labor efectuada. También se viene haciendo hincapié en la mayor visibilidad y difusión de las revistas científicas dentro del ámbito de los profesionales de la psicología tanto a nivel nacional como internacional.

Básicamente, la Asociación Chilena de Revistas Científicas de Psicología tiene como propósito contribuir al fortalecimiento de las publicaciones periódicas en la psicología chilena por medio de un trabajo colaborativo entre revistas, también se mantienen fuertes lazos de cooperación con el mundo de la edición latinoamericana e iberoamericana, lo que se materializa con reuniones de editores en los congresos interamericanos y regionales de la Sociedad Interamericana de Psicología. En la actualidad se puede constatar que la mayoría de las revistas de psicología vigentes se encuentran trabajando de manera coordinada con la asociación. En la
Tabla 4 se puede cotejar la indización de las revistas que son parte de esta Asociación.

De las 9 publicaciones periódicas que son parte de la Asociación Chilena de Revistas Científicas de Psicología, solamente 7 se encuentran incluidas en al menos alguna de las siguientes bases de datos: Redalyc, Scielo, Scopus o Web of Science (WOS). No obstante, Summa Psicológica y Praxis pueden ser recuperadas desde Dialnet. Si se considera la diversidad de los repositorios y bases de datos seleccionadas en la Tabla 4, que a su vez contienen diferentes criterios de inclusión para las revistas científicas (Miguel, 2011), se puede observar que solamente Terapia Psicológica, se encuentra incluida en la reconocida WOS. Por su parte, dos publicaciones generalistas han alcanzado la base Scopus: Psykhe y Psicoperspectivas.

Con todo, es importante señalar que Thomson Reuters ha iniciado un nuevo índex denominado Emerging Sources Citation Index (ESCI), el cual consiste en la valoración por parte de Thomson Reuters a un grupo inicial de 2.400 revistas científicas de 82 países, publicaciones que se encuentran en observación ya que tienen potencial para entrar a las bases de datos principales de la Web of Science, es decir a Science Citation Index (SCI-EXPANDED), Social Sciences Citation Index (SSCI) y Arts y Humanities Citation Index (AyHCI). Al considerar este nuevo subíndice, la única revista de psicología chilena que se encuentra en estado emergente es Cuadernos de Neuropsicología. Se espera que en el futuro no solo nuevas revistas sean consideradas en estado emergente, sino además, muchas de ellas sean incluidas en otros repositorios y bases de datos de carácter internacional. Para ello, las actividades de cooperación entre las diferentes instituciones encargadas de gestionar las publicaciones periódicas y la Asociación Chilena de Revistas Científicas de Psicología se estima esencial.

Tabla 4. Revistas de la Asociación Chilena de Revistas científicas de psicología y su Indexación

\begin{tabular}{|c|c|c|c|c|}
\hline Revista & Redalyc & SciELO & Scopus & WoS \\
\hline Terapia Psicológica & o & o & o & o \\
\hline Psykhe & o & o & o & \\
\hline Psicoperspectivas & o & o & o & \\
\hline Revista de Psicología & o & o & & \\
\hline Cuadernos de Neuropsicología & o & & & \\
\hline Salud \& Sociedad & o & & & \\
\hline Revista Chilena de Neuropsicología & o & & & \\
\hline \multicolumn{5}{|l|}{ Summa Psicológica } \\
\hline Praxis & & & & \\
\hline
\end{tabular}




\section{Consideraciones finales}

Una de las cuestiones que se puede destacar respecto de la evolución histórica de las publicaciones seriadas en psicología en Chile es la poca planificación y visibilidad de las mismas, ya que muchas nacieron y se desarrollaron inicialmente sin un plan estratégico. En su mayoría fueron gestionadas sin tener en cuenta mayores criterios de supervivencia o análisis en cuanto a su calidad editorial, de contenidos, de accesibilidad (desarrollo para web y dispositivos móviles), de visibilidad, de difusión y de sostenibilidad financiera y administrativa. Dicha falta de planificación ha redundado en un final prematuro de una gran mayoría de estos emprendimientos editoriales, y por ende, en la pérdida de inestimables recursos. Cuando se contempla el tiempo promedio de duración de las revistas editadas llama la atención que 13 revistas no sobrepasaron los 2 años de edición, mientras que otras 8 revistas no excedieron los 5 años de publicación continua, lo que en su conjunto indica que un total de 21 revistas se fueron quedando en el camino muy tempranamente.

En general, se puede decir que el trabajo editorial de revistas científicas en psicología en Chile ha estado acompañado por un fuerte desconocimiento o valoración del rol, la responsabilidad y tiempo que supone la labor de la edición científica, el carácter no exclusivo y muchas veces precario de la labor del editor son una prueba de ello. Tampoco se ha tenido demasiada claridad respecto de los objetivos que debería contemplar una publicación periódica, lo que demuestra que la edición científica, no carente de entusiasmo, ha sido más casera que profesional. En este sentido, resulta evidente que para garantizar el éxito y crecimiento de las revistas periódicas en psicología en el país sea necesario tomar medidas explicitas que apunten por un lado a disminuir los efectos de una mala praxis en la edición científica, y por otro lado, fortalezcan buenas prácticas, y a largo plazo, que han demostrado un reducido número de revistas.

Ciertamente la historia de las revistas periódicas en psicología en Chile refleja que muy pocas han logrado mantenerse en el tiempo. No obstante, de esas pocas revistas, varias han logrado crecer y mejorar en términos de calidad y visibilidad, además de alcanzar buenos niveles de indización regional e internacional. Si bien es común creer que lo que evalúan estos sistemas de indización es la calidad de una revista, lo que consideran y valoran es algo mucho más complejo y se relaciona con el ejercicio permanente y sostenido de buenas prácticas editoriales.

Dentro de las líneas de trabajo futuro como editores científicos está el reforzar lazos de cooperación tanto nacionales como internacionales con asociaciones similares, articular puntos en común para dar respuesta a las exigencias en el campo tecnológico y de gestión de contenidos así como sobre la manera en que se evaluará la calidad de sus contenidos. Lo anterior permitirá, por un lado, mejorar la visibilidad e internacionalización de las publicaciones y por otro reconocer y valorar el trabajo del equipo editorial como una labor profesional que requiere de planes de acción concretos y aportes sostenidos en el largo plazo.

Desde luego, todavía hace falta realizar un esfuerzo más categórico para lograr una mejor posición de las revistas chilenas de psicología en el concierto de la ciencia mundial. Si bien esto puede ser una tarea que requiera importantes acciones y consensos entre la comunidad local de editores de revistas de psicología, se considera que la actividad de la Asociación Chilena de Revistas Científicas de Psicología podría servir favorablemente para diseñar y proyectar una política editorial que apunte a la mejora de calidad y visibilidad de las publicaciones periódica en psicología en Chile.

\section{Referencias}

Aguado-López, E., Becerril-García, A., y Aguilar, M. (2016). Universitas Psychologica: un camino hacia la internacionalización. Universitas Psychologica, 15, 321-338. http://dx.doi.org/10.11144/Javeriana. upsy15-2.upci

Alonso Olivas-Ávila, J., Musi-Lechuga, B., Quevedo-Blasco, R., y Luna-Hernández, J. (2012). Índice de Internacionalidad de las revistas iberoamericanas de Psicología en el Journal Citation Reports (2011). Revista Latinoamericana de Psicología, 44, 175-183.

Alarcón, R. (2000). Historia de la psicología en el Perú. De la colonia a la república. Lima: Universidad Ricardo Palma.

Alarcón, R. (2004). Medio siglo de psicología latinoamericana: una visión de conjunto. Revista Interamericana de Psicología/Interamerican Journal of Psychology, 38, 307-316.

Anónimo. (1991). Nota del Director. Revista de Psicología, 26, 7.

Arbaiza-Bayona, A. (2012). Revistas científicas estudiantiles de psicología en Latinoamérica. Revista Colombiana de Psicología, 21, 151-164.

Ardila, R. (1972). La psicología contemporánea. Panorama internacional. Buenos Aires: Paidós.

Ardila, R. (1986). La psicología en América Latina: pasado, presente y futuro. México: Siglo XXI.

Ávila-Toscano, J. H., Marenco-Escuderos, A., y Madariaga, C. (2014). Indicadores bibliométricos, redes de coautorías y colaboración institucional en revistas colombianas de psicología. Avances en Psicología Latinoamericana, 32, 167-182. doi: dx.doi.org/10.12804/apl32.1.2014.12

Benjamin, L T. (1996). The founding of the American Psychologist. The professional journal that wasn 't. American Psychologist, 51, 8-12.

Beyés, R. (2015). Hace medio siglo. Anuario de Psicología, 45, 283-286.

Bizarro, L (2006). Criação da ABECiP. Psicologia: Reflexão e Crítica, 19 , iii.

Blumen, S. (2013). Editorial 30 años de publicación ininterrumpida de la Revista de Psicología. Revista de Psicología, 31, 173-176.

Bravo, L. (2004). Cincuenta años de psicología en la Universidad Católica. Psykhe, 13, 197-204.

Bregman, C., López López, W., y García, F. (2015). La psicología clínica en las publicaciones hispanoamericanas: Un análisis bibliométrico del período 2012-2014. Terapia Psicológica, 33, 205-219. 
Brown, S. (Ed.). (2005). Dictionary of Twentieth-Century British Philosophers. (Vol. 1). Bristol, UK: Theommes.

Buela-Casal, G., y López López, W. (2005). Evaluación de las revistas científicas iberoamericanas de psicología. Iniciativas y estado actual. Revista Latinoamericana de Psicología, 37, 211-217.

Cairo Valcárce, E. (2000). Tres lustros de la Revista Cubana de Psicología. Revista Cubana de Psicología, 17, 3-27.

Calleja, B., y Albornoz, M. (Eds.) (2006). Diálogo entre editores cientificos iberoamericanos. Buenos Aires: CAICYT/CONICET.

Carbonell, X., y Calvó, N. (2009). Las revistas españolas de Psicología: Cómo elegir la revista donde publicar. Anales de psicología, 25, 209216.

Cardoso Sampaio, M. (2008). Citações a periódicos na produção científica de psicologia. Psicologia: Ciência e Profissão, 28, 425-465.

Cardoso Sampaio, M., y Zoqui Paulovic, A. (2012). La merecida visibilidad de las revistas latinoamericanas de psicología. Revista Colombiana de Psicología, 21, 111-123.

Carpintero, H., y Tortosa, F. (1996). La psicología española a través de la Revista de Psicología General y Aplicada. Revista de Psicología General y Aplicada, 49, 373-410.

Carvajal, E., y Matamoros, L. (2012). Análisis bibliométrico de la Revista Costarricense de Psicología, periodo 2001-2011. A propósito de los 30 años de su creación. Revista Costarricense de Psicología, 31, 1-20.

Cassepp, V. (2004). Los 38 años de historia de la Revista Interamericana de Psicología. Revista Interamericana de Psicología/Interamerican Journal of Psychology, 38, 369-372.

Catell, J. (1898). The advances of psychology. Science, 199, 533-541.

Céspedes Alvarado, D. (2008). Una mirada retrospectiva en el 25 aniversario de la Revista Costarricense de Psicología. Revista Costarricense de Psicología, 27, 13-21, 2008

Cetto, A., y Hillerud, K. (Eds.) (1995). Publicaciones científicas en América Latina. México: ICSU/UNESCO/AIC/UNAM/FCE.

Cetto, A., y Alonso, O. (Eds.) (1999). Revistas cientificas en América Latina. México: ICSU/UNAM/CNCT/FCE.

Cetto, A. (2009). Editorial: Calidad e impacto de las revistas iberoamericanas, a debate. Interciencia, 34, 841.

Colotla, V. (2015). Gustave Mark Gilbert: sexto presidente de la Sociedad Interamericana de Psicología. Revista Interamericana de Psicologíal Interamerican Journal of Psychology, 49, 250-260.

Centofanti, R. (1982). Radecki e a psicologia no Brasil. Psicologia: Ciência e Profissão, 3(1), 3-50.

Corredor Aristizábal, J. (2016). Editorial. Revista Colombiana de Psicología, 25, 197-199.

Cueto, M. (1989). Excelencia cientifica en la periferia. Lima: GRADECONCYTEC.

Cruz, N. (2011). Los Anales de la Universidad de Chile y la salud de los chilenos en el siglo XIX. In R. Sagredo. Anales de la Universidad de Chile. Selección de textos médicos. 1857-1887. (pp. ix-lii). Santiago: Cámara Chilena de la Construcción.

Delabarre, E. B. (1895). L'Année psychologique. Première Année 1894, publiee par MM.H Beaunis et A. Binet. Science, 28, 51-53.

Escotet, M. (1978). La psicología en Venezuela. En R. Ardila (Ed.) La profesión del psicólogo (pp. 49-68). México: Trillas.

Fin Motta, R., Gomes de Castro, T., y Pizzinato, A. (2015). A psicologia nos Centros de Referência da Assistência Social (CRAS): um panorama das publicações científicas brasileras de 2004 a 2014. Liberabit, 21, 341-348

Fuchs, A. (2012). 125 years of The American Journal of Psychology. American Journal of Psychology, 125, 3-8.

Gallegos, M. (2009). Movimiento y participación estudiantil en la psicología latinoamericana: consideraciones históricas y futuras perspectivas. Avances en Psicología Latinoamericana, 27, 33-60.

Gallegos, M. (2010). La Revista Latinoamericana de Psicología en sus 40 años de historia: 1969-2009. Universitas Psychologica, 9, 911-924.

Gallegos, M. (2013). Sixty years of the Interamerican Society of Psychology: origins and developments. International Journal of Psychology, $48,1313-1320$
Gallegos, M. (2014a). La génesis de la profesión psicológica. Eureka. Revista Cientifica de Psicología, 11, 134-148.

Gallegos, M. (2014b). La psicología de los menores en América del Sur: historia del movimiento estudiantil y escenarios futuros. In G. Salas (Ed.), Historias de la psicología en América del Sur. Diálogos y perspectivas (pp. 278-305). La Serena, Chile: Mirada.

Gallegos, M. (en prensa). La Revista Cubana de Psicología de 1955: una historia previa a la revolución. Revista de Psicología, 26.

Gallegos, M., Berra, M., Benito, E., y López López, W. (2014). Las nuevas dinámicas del conocimiento científico y su impacto en la psicología Latinoamericana. Psicoperspectivas, 13, 106-117.

Gamba, E., Packer, A., y Meneghini, R. (2015). Pathways to internationalize brazilian journals of psychology. Psicologia: Reflexão e Crítica, 28, 66-71. doi: http://dx.doi.org/10.1590/1678-7153.20152840010

Garcia, A., Acevedo-Triana, C., y López-López, W. (2014) Cooperación en las Ciencias del Comportamiento Latinoamericanas: Una Investigación Documental. Terapia Psicológica, 32, 165-174. DOI: https://dx.doi. org/10.4067/S0718-48082014000200009

García, J. (2016). La tercera Jornada de Estudiantes de Psicología en Asunción (1983). Nuevos Paradigmas, 1, 9-20.

García Martínez, A., y Guerrero Bote, V. (2008). La psicología en el cienciograma de los países iberoamericanos. Revista Latinoamericana de Psicología, 40, 409-424.

García Martínez, A., Guerrero Bote, V., y De Moya Anegon, F. (2012). World Scientific production in psychology. Universitas Psychologica, 11, 669-717.

Gibbons, M., Limoges, C., Nowotny, H., Schwartzman, S., Scott, P., y Trow, M. (1997). La nueva producción del conocimiento. Barcelona: Pomares-Corredor.

Green, C., Feinerer, I., y Burman, JT. (2015). Searching fot the structure of early American Psychology: Networking Psychological Review, 1894-1908. History of Psychology, 18, 15-31. doi: 10.1037/a0038406

Green, C., y Feinerer, I. (2015). The evolution of The American Journal of Psychology 1, 1887-1903: A network investigation. American Journal of Psychology, 128, 387-401. doi: 10.5406/amerjpsyc.128.3.0387

Green, C., y Feinerer, I. (2016). The evolution of The American Journal of Psychology 2, 1904-1918: A network investigation. American Journal of Psychology, 129, 185-196. doi: 10.5406/amerjpsyc.129.2.0185

Gómez-Morales, Y., Jaraba-Barrios, B., Guerrero-Castro, J., y LópezLópez, W. (2012). Entre internacionalización y consolidación de comunidades académicas locales. Sobre la Revista Latinoamericana de Psicología. Revista Colombiana de Psicología, 21, 97-110.

Guárdia Olmos, R. (2015). Editorial. Anuario de Psicología, 45, 281-283.

Gutiérrez, G., Pérez-Acosta, A., y Plata-Caviedes, T. (2009). Desarrollo histórico de una publicación científica: cuarenta años de la Revista Latinoamericana de Psicología. Revista Latinoamericana de Psicología, 41, 413-428.

Haupt, C. (2000). Las revistas científicas latinoamericanas: su difusión y acceso a través de bases de datos. Biblioteca Universitaria, 3, 122-127.

Jacó-Vilela, A. (1999). Arquivos Brasileiros de Psicotécnica e Boletim do Instituto de Psicologia: Psicologia no Brasil. En M. Guedes y R. Campos (Eds.), Estudos em história da psicologia (pp. 119-135). São Paulo: Educ.

Kaulino, A. (2016). "Más vale prevenir que curar y reeducar que reprimir": Características psicosociales de los niños chilenos abandonados y delincuentes, según Abelardo Iturriaga. Revista de Psicología, 25, 1-7. doi: http://dx.doi.org/10.5354/0719-0581.2016.44583

Klappenbach, H., y Arrigoni, F. (2011). Revista Argentina de Psicología. 1969-2002. Estudio bibliométrico. Revista Argentina de Psicología, 50, 44-94.

Klappenbach, H., y Pavesi, P. (1994). Una historia de la psicología en Latinoamérica. Revista Latinoamericana de Psicología, 26, 445-482.

Laborda, M., y Quezada, V. (Eds.) (2010). Notas históricas de la psicología en Chile. Santiago, Chile: Editorial Universitaria.

Lafarga, J. (1984). Editorial. Revista Mexicana de Psicología, 1, 3-4.

León, R. (1997). Rumbo al nuevo mundo: cuatro psicólogos de Europa Oriental en la historia de la psicología en América del Sur. Revista Latinoamericana de Psicología, 29, 9-34. 
León, R. (2014). Notas acerca de psicólogos y teorías psicológicas de Europa Oriental en la historia de la psicología en América del Sur. Liberabit, 20, 55-72.

Ligüeño, S., y Parra, D. (2007). La psicología en la Universidad de Chile: una propuesta de estudio para comprender la historia de la psicología en Chile. Cuadernos de Neuropsicología, 1, 223-235.

López, J. (2000). La primera revista médica de América. ACIMED, 8, 1339-139.

López Caicedo, B. (1992). Presentación. Revista Colombiana de Psicología, 1,5 .

López-López, W. (2010). Gestión de conocimiento y redes: la nueva red de editores de revistas de psicología de Iberoamérica. Universitas Psychologica, 9, 607.

López-López, W. (2014). Editorial: La publicación científica los compromisos de las comunidades de investigadores. Universitas Psychologica, 13, 326-326.-421.

López-López, W. (2015). Changes in scientific output in psychology in Iberoamerica in the last decade. Universitas Psychologica, 14, 1.

López-López, W., de Moya Anegón, F., AcevedoTriana, C., Garcia, A., y Silva, L. M. (2015). Visibility and Cooperation in Iberoamerican Psychology. Psicologia: Reflexão e Crítica, 28, 72-81. doi: http://dx.doi. org/10.1590/1678-7153.20152840011

Luco, A. (2010). Psicología en Chile. Ciencia, disciplina y profesión. Santiago: Catalonia.

Luna-Morales, M., y Collazo-Reyes, F. (2007). Análisis histórico bibliométrico de las revistas latinoamericanas y caribeñas en los índices de la ciencia internacional: 1961-2005. Revista Española de Documentación Científica, 30, 523-543.

Marín, G. (1985). Difusión internacional de la psicología iberoamericana: dimensiones del problema. Revista Mexicana de Psicología, 2, 12-19.

Martínez-Taboas, A., y Pérez Pedrogo, C. (2006). Las revistas de psicología en Puerto Rico: Una breve mirada histórica. Revista Puertorriqueña de Psicología, 17, 571-589.

Miguel, S. (2011). Revistas y producción científica de América Latina y el Caribe: su visibilidad en SciELO, RedALyC y SCOPUS. Revista Interamericana de Bibliotecología, 34, 187-199.

Miguel, S., Tannuri de Oliveira, E., y Cabrini, H. (2016). Scientific production on open access: A worldwide bibliometric analysis in the academic and scientific context. Publications, 4, 1- 15. Doi: 10.3390/ publications 4010001

Montenegro, M., Pujol, J., y Vargas-Monroy, L. (2015). Miradas, formas de hacer y relaciones en la constitución de una investigación crítica. Universitas Psychologica, 14, 1833-1852.

Montoya, E. (2012). La investigación científica en Iberoamérica (19822011). Estudio basado en la bibliometría. Revista Iberoamericana de Ciencia, Tecnología y Sociedad, 21, 63-79.

Nascimento, J. (1924). Editorial. Atenea, 1, 3-5.

Naidorf, J. (2009). Los cambios en la cultura académica de la universidad pública. Buenos Aires: Eudeba.

Newcomb, S. (1906). The organization of scientific research. The North American Review, 182, 32-43.

Nicolas, S., Segui, J., y Ferrand, L. (2000). Les premières revues de psychologie: la place de "L'Année Psychologique". L'Année Psychologique, 100, 71-110. doi: 10.3406/psy.2000.28628

Peñaranda Ortega, M., Quiñones Vidal, E., y López García, J. (2005). Veinte años de Anales de Psicología: Una revista con raíces académicas (1984-2004). Anales de psicología, 21, 181-198.

Parra, D. (2015). Un análisis historiográfico sobre las relaciones entre psicología y educación en Chile (1889-1973). Revista de Historia de la Psicología, 36, 95-114.

Polanco-Carrasco, R. (2015). Editar para perturbar. Cuadernos de Neuropsicología, 9, 10-11. doi: 10.7714/cnps/9.3.101

Polanco-Carrasco, R. (2016a). La publicación científica como placebo. Cuadernos de Neuropsicología, 10, 8-12. doi: 10.7714/CNPS/10.2.101

Polanco-Carrasco, R. (2016b). ¿Por qué llevase un paper a la cama? Cuadernos de Neuropsicología, 10, 10-12. doi: 10.7714/CNPS/10.3.101

Price, D. (1973). Hacia una ciencia de la ciencia. Barcelona: Ariel.
Pérez-Acosta, A. (2003). Red de editores de revistas psicológicas colombiana. Revista Electrónica Hispanoamericana de Psicología, 49,1 .

Quevedo-Blasco, R. (2013). Revistas iberoamericanas de Psicología indexadas en el Journal citations Reports de 2011. Revista Mexicana de Psicología, 30, 1-10.

Quevedo-Blasco, R. (2015). Análisis de las revistas iberoamericanas de Psicología y de Educación indexadas en el Journal Citation Reports del 2013. European Journal of Education and Psychology, 8, 85-96.

Quevedo-Blasco, R., y Ariza, T. (2013). Twenty-fi ve year history of the journal Psicothema. Psicothema, 25, 93-99. doi: 10.7334/psicothema2012.282

Quevedo-Blasco, R., y López López, W. (2010). Análisis bibliométrico de las revistas multidisciplinares de psicología recientemente incorporadas en la Web of Science (2008-2009). Psicologia: Reflexão e Crítica, 23, 384-408.

Quevedo-Blasco, R., y López López, W. (2011). Situación de las revistas iberoamericanas de psicología en el Journal Citation Reports de 2010. Universitas Psychologica, 10, 937-947.

Rodríguez, P., y Sánchez, L. (1999). La psicología en Venezuela. En M. Alonso y A. Eagly (Eds.), Psicología en las Américas (pp. 307-316). Buenos Aires: SIP.

Romero Croce, J. (2014). Estudio bibliométrico de la revista Liberabit en el período comprendido entre 1995-2013. Liberabit, 20, 368-372.

Romero Medina, A. (2009). Evolución de la calidad y difusión de una revista científica española de Psicología: Anales de Psicología, 25 volúmenes anuales publicados (1984-2009). Anales de Psicología, 25, 181-198.

Salas, G. (2013). Juan Serapio Lois (1844-1913). Pionero de la psicología científica en Chile. Psykhe, 22, 111-123. doi: 10.7764/psykhe.22.630

Salas, G. (2014). El Informe Nassar (1955) sobre la formación de psicólogos en Chile. Revista de Psicología, 23, 109-112.

Salas, G. (2015). La Revista Mente (1920-1922) como contraparte de la psicología científica en Chile. Actas II Congreso Anual de la Sociedad Científica de Psicología. 8 y 9 de enero. Santiago: Sociedad Científica de Psicología de Chile.

Salas, G., y Lizama, E. (2013). Historia de la psicología en Chile: 18891981. (2 ${ }^{\circ}$ Ed.). La Serena: Universidad de La Serena.

Sherrington, C.S. (1922). The maintenance of scientific research. Science, $55,629-631$.

Staley, T. W. (2009). The Journal Mind in its early years, 1876-1920: An introdiuction. Journal of the history of ideas, 70, 259-263.

Sanz Ferramola, R., y Klappenbach, H. (2000). La psicología en los años treinta: estudio bibliométrico de los Anales del Instituto de Psicología (1935-1941). En J. Ríos, R. Ruiz, J. Stagnaro y P. Weissmann (Eds.), Psiquiatría, psicología y psicoanálisis. Historia y memoria (pp. 269284). Buenos Aires: Polemos.

Sampaio, I., y Zoqui, A. A. (2012). La merecida visibilidad de las revistas latinoamericanas de psicología. Revista Colombiana de Psicología, $21,109-121$.

Subramanyan, K. (1983). Bibliometric studies of research collaboration. Journal of Information Science. 6, 33-38.

Stanley Hall, G., y Titchener, E. (1921). The American Journal of Psychology, 32, 1-4.

Titchener, E. (1921). Wilhelm Wundt. The American Journal of Psycho$\log y, 32,161-178$

Toro, J., y Villegas, J. (1999). Psicología en Chile. En M. Alonso y A. Eagly (Eds.), Psicología en las Américas (pp. 129-153) Buenos Aires: SIP.

Trzesniak, P., Plata-Caviedes, T., y Córdoba-Salgado, O. (2012). Qualidade de conteúdo, o grande desafio para os editores científicos. Revista Colombiana de Psicologia, 21, 57-75.

Urzúa, A. (2008). Elementos contextuales a la investigación en psicología en Chile. Revista Interamericana de Psicología/Interamerican Journal of Psychology, 42, 446-461.

Urzúa, A., Vera-Villarroel, P., Zúñiga, C., y Salas, G. (2015). La psicología en Chile: análisis de su historia, presente y futuro. Universitas Psychologica, 14, 1125-1142. doi: http://dx.doi.org/10.11144/Javeriana. upsy14-3.pcah 
VandenBos, G., y Winkler, J. (2015). An analysis of the status of journals and research in psychology from Latin America. Psicologia: Reflexão e Crítica, 28, 82-93. doi: http://dx.doi.org/10.1590/16787153.20152840012

Valderrama, J.O. (2012). Publicar en revistas cientñificas de corriente principal: Antecedentes, definiciones y recomendaciones. La Serena: Universidad de La Serena.

Vera-Villarroel, P., y Lillo, S. (2006). La investigación actual en psicología clínica en Chile: un análisis a partir de la producción. Terapia Psicológica, 24, 221-230.

Vera-Villaroel, P., López López, W., Lillo, S., y Silva, M. (2011). La producción científica en psicología latinoamericana: Un análisis de la investigación por países. Revista Latinoamericana de Psicología, 43, 95-104.

Vessuri, H. (1983). La ciencia periférica. Caracas: Monte Ávila.

Woodworth, R.S. (1909). Hermann Ebbinghaus. The Journal of Philosophy, Psychology and Scientific Methods, 6, 253-256.

Zych, I., y Buela-Casal, G. (2010). Internacionalidad de las revistas de psicología multidisciplinar editadas en Iberoamérica. Universitas Psychologica, 9, 27-34. 
